\title{
Association between dietary factors and plasma fetuin-A concentrations in the general population
}

\author{
Katharina Nimptsch ${ }^{1 *}$, Jürgen Janke ${ }^{1}$, Tobias Pischon ${ }^{1}$ and Jakob Linseisen ${ }^{2}$ \\ ${ }^{1}$ Molecular Epidemiology Research Group, Max Delbrück Center for Molecular Medicine (MDC), 13125 Berlin, Germany \\ ${ }^{2}$ Institute of Epidemiology 2, Helmboltz Centre Munich, 85764 Neuberberg, Germany \\ (Submitted 27 January 2015 - Final revision received 21 May 2015 - Accepted 18 June 2015 - First published online 28 August 2015)
}

\section{Abstract}

Circulating fetuin-A, a novel marker for hepatic fat accumulation, has been related to a higher risk of type 2 diabetes and cardiovascular diseases in a growing number of prospective studies. However, little is known about dietary determinants of fetuin-A concentrations in the general population. Therefore, we aimed to investigate the association between dietary intake of energy, energy-providing nutrients, alcohol and major food groups and plasma fetuin-A concentrations in the Bavarian Food Consumption Survey II. Dietary intake was assessed by three 24-h dietary recalls, and plasma concentrations of fetuin-A were measured in 558 adults (18-81 years). After multivariable adjustment for lifestyle factors and body fatness, higher energy intake was nonsignificantly associated with higher fetuin-A concentrations (per $2092 \mathrm{~kJ} / \mathrm{d}$ $(500 \mathrm{kcal} / \mathrm{d}) \quad 3.7 \mu \mathrm{g} / \mathrm{ml}, 95 \% \mathrm{CI}-0.5,7.8 \mu \mathrm{g} / \mathrm{ml})$. There was no clear association between energy-providing nutrients and fetuin-A concentrations. Higher alcohol intake was associated with lower fetuin-A concentrations ( $\left.P_{\text {trend }} 0 \cdot 003\right)$ : mean fetuin-A concentrations were 324 $(95 \%$ CI 313, 335) $\mu \mathrm{g} / \mathrm{ml}$ in non-drinkers, and with $293(95 \%$ CI 281, 306) $\mu \mathrm{g} / \mathrm{ml}$ significantly lower in participants who drank $\geq 30 \mathrm{~g}$ alcohol per $\mathrm{d}$. Mean fetuin-A concentrations decreased across quintiles of milk and dairy product intake (lowest quintile 319 (95\% CI 309, 330) $\mu \mathrm{g} / \mathrm{ml}$; highest quintile $\left.304(95 \% \mathrm{CI} 293,314) \mu \mathrm{g} / \mathrm{ml} ; P_{\text {trend }} 0.03\right)$, and each 150-g increment in milk/dairy products per d was associated with $5.6(95 \%$ CI $-9 \cdot 6,-1 \cdot 5) \mu \mathrm{g} / \mathrm{ml}$ lower fetuin-A. Dietary intakes of vegetables, meat or fish were not associated with fetuin-A concentrations. Because of the preventive potential of our findings, further exploration is warranted.

\section{Key words: Fetuin-A: Dietary intake: Energy intake: Alcohol: Dairy products}

Fetuin-A, also referred to as $\alpha 2$-Heremans-Schmid glycoprotein (AHSG), is a protein synthesised and secreted by the liver, particularly in hepatic steatosis ${ }^{(1)}$. To a lesser degree, fetuin-A is also secreted by placenta and tongue, and recent findings suggest that it is also expressed and secreted by adipose tissue $^{(1,2)}$. Fetuin-A is related to hepatic insulin resistance and subclinical inflammation, and it has been suggested as a novel marker for hepatic fat accumulation ${ }^{(1,3,4)}$. Mice deficient for the AHSG gene are resistant to weight gain upon a high-fat diet ${ }^{(5,6)}$. Observational studies have shown a positive association between fetuin-A and obesity ${ }^{(3,7)}$, and a recent bidirectional Mendelian Randomisation study suggests that fetuin-A is causally associated with higher $\mathrm{BMI}^{(8)}$. In addition, there is growing evidence from prospective studies that high plasma fetuin-A concentrations are associated with a higher risk of type 2 diabetes $^{(4,9)}$ and cardiovascular diseases ${ }^{(7)}$. Taken together, fetuin-A has a role in a number of metabolic conditions and chronic diseases. Therefore, knowledge of modifiable determinants of circulating fetuin-A has direct public health relevance. However, so far little is known about dietary determinants of
fetuin-A concentrations in the general population. In a randomised clinical trial among 76 overweight diabetic women, calorie restriction resulted in a decrease in fetuin-A concentrations ${ }^{(10)}$. In a cross-sectional analysis within a subcohort from the European Prospective Investigation into Cancer and Nutrition (EPIC)-Potsdam Study, dietary intake of red meat or whole grain was not associated with fetuin-A concentrations ${ }^{(11)}$. In a recent analysis of women from the Nurses' Health Study, an inverse association between alcohol consumption and fetuin-A concentrations was observed, and fetuin-A explained a substantial proportion of the inverse association between alcohol consumption and risk of type 2 diabetes ${ }^{(12)}$. In both the EPIC-Potsdam Study and the Nurses' Health Study, dietary intake was assessed using FFQ.

With this study, we aimed to investigate the association between energy intake, energy-providing nutrients, alcohol consumption and major food groups and plasma fetuin-A concentrations in the Bavarian Food Consumption Survey II (BVS II), a population-based survey in which dietary intake was assessed by three 24-h dietary recalls.

\footnotetext{
Abbreviation: BVS II, Bavarian Food Consumption Survey II.
}

* Corresponding author: Dr K. Nimptsch, fax + 49309406 4576, email katharina.nimptsch@mdc-berlin.de 


\section{Methods}

\section{Study design and population}

The BVS II is a cross-sectional study designed to be representative for the Bavarian population. The aim of the study was to investigate dietary and lifestyle habits in Bavaria. The study population comprises 1050 German-speaking participants aged 13-80 years who were recruited in a three-stage random route sampling procedure between September 2002 and June 2003. Participants' characteristics, lifestyle factors and medical history were collected during a computer-aided personal interview. Within 2 weeks after recruitment, trained interviewers contacted participants by telephone three times ( 2 weekdays and 1 weekend day) to assess dietary intake by 24-h dietary recalls. In addition to dietary information, physical activity on the previous day was assessed at the end of each telephone interview using standardised questions on type and duration of physical activity, as well as duration of television/personal computer time and sleeping hours. The overall participation rate was $71 \%$. Within 6 weeks after recruitment, all adults ( $\geq 18$ years) who had completed the baseline interview and at least one dietary recall were invited to their nearest public health office for blood sampling and anthropometrical measurements. Out of 879 invited subjects, 568 persons followed the invitation ( $65 \%$ of eligible persons). The study was conducted according to the guidelines laid down in the Declaration of Helsinki, and all procedures involving human subjects were approved by the local ethics committee (Bavarian Ministry of Health) ${ }^{(13)}$. Written informed consent was obtained from all study participants.

\section{Dietary intake assessment}

The 24-h dietary recalls were conducted using the EPIC-Soft software ${ }^{(14,15)}$. The data from the three recalls per person were weighted for weekday and weekend day to calculate the average daily food intake. The different foods reported during the 24-h dietary recalls were grouped into seventeen food groups. Nutrient intakes were calculated using food content data from the German food composition database 'Bundeslebensmittelschluessel' (version II.3) ${ }^{(16)}$

\section{Blood sampling and laboratory measurements}

Venous blood was drawn into EDTA tubes or serum tubes, chilled at $4^{\circ} \mathrm{C}$ and processed subsequently within $3 \mathrm{~h}$. Serum was separated from blood cells by centrifugation, and samples were divided into aliquots. Samples were cooled for a maximum of $1 \mathrm{~d}$ until they were stored at $-80^{\circ} \mathrm{C}$. Plasma concentrations of fetuin-A were measured by ELISA (BioVendor Human Fetuin-A ELISA; intra-assay CV 3.9-6.5\%, inter-assay CV $2 \cdot 6-5 \cdot 1 \%$ according to the manufacturer) in the laboratory of Professor Pischon, Molecular Epidemiology Group, Max Delbrück Center for Molecular Medicine (MDC). On the basis of internal quality control samples (two on each of the eight analysis plates), the inter-assay $\mathrm{CV}$ was $7.7 \%$.

\section{Statistical analysis}

After exclusion of participants with missing information on diet $(n$ 7) or fetuin-A ( $n$ 3), 558 participants (235 men and 323 women) were included in the statistical analysis. Waist circumference was missing for a few $(n 8)$ study participants. For statistical analysis, these missing values were replaced with sex-specific median values. In descriptive statistics, we compared participants' characteristics across sex-specific quintiles of fetuin-A concentrations. The association between total energy intake, energy-providing nutrients (dietary fat, carbohydrates, protein), alcohol or major food groups (vegetables, fruit, milk/dairy products, unprocessed red meat, processed meat, poultry and fish) and fetuin-A concentrations was investigated using multivariable linear regression models with robust variance ${ }^{(17)}$. Results are presented as mean fetuin-A concentrations with corresponding $95 \%$ confidence intervals in quintiles or categories of dietary intake. In addition, continuous estimates showing the increase or decrease in fetuin-A associated with a prespecified increment in dietary intake are presented. Multivariable models were adjusted for age (continuous in years), sex, smoking status (never, former, current), social status (five categories), physical activity (sex-specific quintiles of total activity in MET-h/d), alcohol intake (except for models investigating alcohol intake; non-drinker or continuous in $\mathrm{g} / \mathrm{d}$ ) and non-alcohol energy intake (continuous in $\mathrm{kcal} / \mathrm{d}$ with and without additional adjustment for BMI (continuous, $\mathrm{kg} / \mathrm{m}^{2}$ ) and residuals of BMI-adjusted waist circumference (to avoid multicollinearity; continuous). Fasting status was not included in the multivariable models because it was not related to fetuin-A concentrations, and inclusion of fasting status into the models did not alter parameter estimates of dietary variables substantially. For the analysis of dietary fat, carbohydrates or protein intake, we created multivariable energy-density models with nutrient intake expressed in percentage of total energy intake and mutual adjustment for energy intake. The continuous estimates from these models estimate the change in circulating fetuin-A associated with a 5\% higher energy intake provided by the nutrient under study in substitution with $5 \%$ of energy provided by carbohydrates (or fat). Because of the relatively large proportion of non-drinkers (10\% in men and $19 \%$ in women), for the analysis of the association between alcohol consumption and fetuin-A we present adjusted mean fetuin-A concentrations in established categories for alcohol intake ${ }^{(18)}$. Similarly, because of the low consumption of poultry and fish, mean fetuin-A concentrations are presented by categories of these two variables (non-consumers, $</ \geq 40 \mathrm{~g} / \mathrm{d}$, which corresponds to the approximate median cut-offs). Tests for linear trends across dietary intake quintiles or categories were performed by modelling the median values in each quintile/category and evaluating this variable's statistical significance using the Wald's test. To correct for multiple hypothesis testing, we took the falsediscovery rate into account, counting each dietary factor under investigation as an independent hypothesis test ${ }^{(19)}$ ( $n$ 12). In prespecified subgroup analyses, we tested for statistical interaction in the association between dietary factors and fetuin-A by sex using cross-product terms. Because no statistically significant interactions by sex were observed (all $P$-values $>0 \cdot 2$ ), only combined associations in the whole study population are presented. However, because under-reporting of energy intake has been shown to depend on sex and $\mathrm{BMI}^{(20,21)}$, we also describe the association between energy intake and fetuin-A concentrations stratified by sex and BMI $\left(</ \geq 25 \mathrm{~kg} / \mathrm{m}^{2}\right)$. 
Table 1. Basic characteristics by quintiles* of circulating fetuin-A in 558 men and women who participated in the Bavarian Food Consumption Survey II (Mean values and standard deviations; numbers and percentages)

\begin{tabular}{|c|c|c|c|c|c|c|c|c|c|c|}
\hline & \multicolumn{2}{|c|}{ Quintile 1} & \multicolumn{2}{|c|}{ Quintile 2} & \multicolumn{2}{|c|}{ Quintile 3} & \multicolumn{2}{|c|}{ Quintile 4} & \multicolumn{2}{|c|}{ Quintile 5} \\
\hline & $n$ & $\%$ & $n$ & $\%$ & $n$ & $\%$ & $n$ & $\%$ & $n$ & $\%$ \\
\hline$N$ & 111 & & 112 & & 112 & & 112 & & 111 & \\
\hline Female sex & 64 & $57 \cdot 7$ & 65 & 58 & 65 & 58 & 65 & 58.0 & 64 & 57.7 \\
\hline \multicolumn{11}{|l|}{ Age (years) } \\
\hline Mean & \multirow{2}{*}{\multicolumn{2}{|c|}{$\begin{array}{l}50 \cdot 4 \\
13 \cdot 8\end{array}$}} & \multirow{2}{*}{\multicolumn{2}{|c|}{$\begin{array}{l}49 \cdot 3 \\
15 \cdot 9\end{array}$}} & \multirow{2}{*}{\multicolumn{2}{|c|}{$\begin{array}{l}51.2 \\
15.9\end{array}$}} & \multicolumn{2}{|c|}{$46 \cdot 8$} & \multicolumn{2}{|c|}{43.5} \\
\hline SD & & & & & & & & & & \\
\hline Current smoking & 60 & $54 \cdot 1$ & 58 & $51 \cdot 8$ & 60 & $53 \cdot 6$ & 61 & 54.5 & 52 & $46 \cdot 8$ \\
\hline Physical activity (MET/h per d) & $13 \cdot 4$ & $13 \cdot 6$ & $10 \cdot 4$ & 8.4 & $11 \cdot 4$ & $8 \cdot 8$ & $12 \cdot 2$ & 8.9 & $11 \cdot 6$ & $10 \cdot 4$ \\
\hline \multicolumn{11}{|l|}{ Waist circumference $(\mathrm{cm})$} \\
\hline Mean & \multicolumn{2}{|c|}{$92 \cdot 4$} & \multicolumn{2}{|c|}{$94 \cdot 6$} & \multicolumn{2}{|c|}{$95 \cdot 8$} & \multicolumn{2}{|c|}{$93 \cdot 9$} & \multicolumn{2}{|c|}{94.9} \\
\hline SD & \multicolumn{2}{|c|}{$14 \cdot 6$} & \multicolumn{2}{|c|}{14.4} & \multicolumn{2}{|c|}{$14 \cdot 6$} & \multicolumn{2}{|c|}{$12 \cdot 5$} & \multicolumn{2}{|c|}{14.6} \\
\hline \multicolumn{11}{|l|}{ BMI $\left(\mathrm{kg} / \mathrm{m}^{2}\right)$} \\
\hline Mean & \multirow{2}{*}{\multicolumn{2}{|c|}{$\begin{array}{r}26.0 \\
4.9\end{array}$}} & \multirow{2}{*}{\multicolumn{2}{|c|}{$\begin{array}{r}26.5 \\
4.8\end{array}$}} & \multirow{2}{*}{\multicolumn{2}{|c|}{$\begin{array}{r}27.3 \\
5.3\end{array}$}} & \multirow{2}{*}{\multicolumn{2}{|c|}{$\begin{array}{r}26.9 \\
4.6\end{array}$}} & \multirow{2}{*}{\multicolumn{2}{|c|}{$\begin{array}{r}26.6 \\
4.9\end{array}$}} \\
\hline SD & & & & & & & & & & \\
\hline Obese & 17 & $15 \cdot 3$ & 26 & $23 \cdot 2$ & 23 & $20 \cdot 5$ & 21 & $18 \cdot 8$ & 23 & 20.7 \\
\hline Prevalent diabetes & 10 & $9 \cdot 0$ & 9 & $8 \cdot 0$ & 10 & 8.9 & 4 & 3.6 & 4 & 3.6 \\
\hline Prevalent hypertension & 28 & $25 \cdot 2$ & 26 & $23 \cdot 2$ & 36 & $32 \cdot 1$ & 25 & $22 \cdot 3$ & 23 & $20 \cdot 7$ \\
\hline
\end{tabular}

* Quintile cut-offs were 264, 291, 314 and $344 \mu \mathrm{g} / \mathrm{ml}$ in men and 265, 299, 325 and $358 \mu \mathrm{g} / \mathrm{ml}$ in women.

In addition, to investigate the robustness of observed associations, we conducted sensitivity analyses. First, we excluded participants who were suspected to be under-reporters of dietary intake based on a low ratio of energy intake (EI) to estimated basal metabolic rate (BMR) (EI/BMR $<0 \cdot 8, n 18$ men, $n 28$ women $)^{(22)}$. Second, we excluded study participants with metabolic diseases ( $n$ 203) that is, participants who were obese (BMI $\left.\geq 30 \mathrm{~kg} / \mathrm{m}^{2}, n 110\right)$ and/ or had reported prevalent diabetes ( $n$ 37) or hypertension ( $n$ 138).

\section{Results}

Mean fetuin-A concentrations were $303.7 \mu \mathrm{g} / \mathrm{ml}$ in men, ranging from 177.6 to $462.7 \mu \mathrm{g} / \mathrm{ml}$, and in women the mean was $314.7 \mu \mathrm{g} / \mathrm{ml}$, with a range from 182.4 to $531.9 \mu \mathrm{g} / \mathrm{ml}$. The mean age of study participants decreased across quintiles of fetuin-A concentrations (Table 1). Waist circumference and BMI slightly increased across fetuin-A quintiles. Mean dietary intakes of energy, alcohol, energy-providing nutrients and major food groups in male and female BVS II participants are shown in online Supplementary Table S1.

There was no clear association between energy intake or intake of energy-providing nutrients with fetuin-A concentrations (Table 2). We observed a slight suggestion of a positive association between total energy intake and circulating fetuin-A (per $2092 \mathrm{~kJ} / \mathrm{d}(500 \mathrm{kcal} / \mathrm{d}$ ) $3.7 \mu \mathrm{g} / \mathrm{ml}, 95 \% \mathrm{CI}-0.5,7.8 \mu \mathrm{g} / \mathrm{ml}$ in the multivariable model including body fatness), but the adjusted mean values across quintiles were not suggestive of a linear trend ( $\left.P_{\text {trend }} 0 \cdot 16\right)$. The continuous estimate was slightly higher but still statistically nonsignificant $(4.5 \mu \mathrm{g} / \mathrm{ml}, 95 \%$ CI -0.3 , $9 \cdot 2 \mu \mathrm{g} / \mathrm{ml}$ ) when under-reporters of energy intake were excluded (sample size after exclusion $n$ 512). Although a statistically significant association between energy intake and fetuin-A concentrations was observed in men (continuous estimate $6.4 \mu \mathrm{g} / \mathrm{ml}, 95 \%$ CI $\left.1.2,11.5 \mu \mathrm{g} / \mathrm{ml}, P_{\text {trend }} 0.02\right)$, there was no association in women $(2.2 \mu \mathrm{g} / \mathrm{ml}, 95 \%$ CI $-4.6,9 \cdot 1 \mu \mathrm{g} / \mathrm{ml}$, $\left.P_{\text {trend }} 0.52 ; P_{\text {interaction }} 0.84\right)$. After stratification by BMI, we observed a statistically significant continuous estimate $(7.9 \mu \mathrm{g} / \mathrm{ml}, 95 \%$ CI $0 \cdot 1,15 \cdot 6 \mu \mathrm{g} / \mathrm{ml})$ and trend across quintiles $\left(P_{\text {trend }} 0.02\right)$ in lean study participants $\left(\mathrm{BMI}<25 \mathrm{~kg} / \mathrm{m}^{2}\right)$, but not in overweight participants (BMI $\geq 25 \mathrm{~kg} / \mathrm{m}^{2}$, continuous estimate $2 \cdot 0 \mu \mathrm{g} / \mathrm{ml}, 95 \%$ CI $\left.-2 \cdot 8,6.7 \mu \mathrm{g} / \mathrm{ml}, P_{\text {trend }} 0 \cdot 87\right)$, although no multiplicative interaction was observed $\left(P_{\text {interaction }} 0 \cdot 40\right)$. Mean fetuin-A concentrations increased slightly across quintiles of fat intake and decreased slightly across quintiles of carbohydrate or protein intake. However, there was no suggestion of any important association between energy-providing nutrient intake and fetuin-A concentrations.

We observed an inverse association between alcohol consumption and fetuin-A concentrations (Table 3). In the multivariable model including body fatness, the mean fetuin-A concentrations were 324 (95\% CI 313,335$) \mu \mathrm{g} / \mathrm{ml}$ in nondrinkers, $311(95 \%$ CI 302, 320) $\mu \mathrm{g} / \mathrm{ml}$ in individuals with low alcohol consumption ( $<5 \mathrm{~g} / \mathrm{d}), 314(95 \%$ CI 304, 323) $\mu \mathrm{g} / \mathrm{ml}$ in individuals with low-moderate alcohol consumption $(5-14.9 \mathrm{~g} / \mathrm{d})$, $303(95 \%$ CI 292, 314) $\mu \mathrm{g} / \mathrm{ml}$ in moderate drinkers $(15-29.9 \mathrm{~g} / \mathrm{d})$ and $293(95 \%$ CI 281,306$) \mu \mathrm{g} / \mathrm{ml}$ in individuals in the highest alcohol consumption category ( $\geq 30 \mathrm{~g} / \mathrm{d}$ ); a statistically significant trend across alcohol intake categories was observed ( $P_{\text {trend }}$ 0.003). The inverse association remained statistically significant after taking the false-discovery rate into account (adjusted $P_{\text {trend }}$ 0.04). The adjusted mean fetuin-A values across alcohol intake categories (data not shown) were similar in men ( $P_{\text {trend }}$ 0.04) and in women $\left(P_{\text {trend }} 0.04\right)$, in lean participants $\left(\mathrm{BMI}<25 \mathrm{~kg} / \mathrm{m}^{2}, P_{\text {trend }} 0.66\right)$ and in overweight $\left(\mathrm{BMI} \geq 25 \mathrm{~kg} / \mathrm{m}^{2}\right.$, $P_{\text {trend }}$ 0.05) participants, and after exclusion of participants with metabolic diseases $\left(P_{\text {trend }} 0.003\right)$.

The associations between dietary intake of major food groups and circulating fetuin-A are shown in Table 4: We observed a statistically significant inverse association between milk and dairy product intake and circulating fetuin-A: mean fetuin-A concentrations decreased across quintiles of milk and dairy products $\left(P_{\text {trend }} 0.02\right.$ ), and each $150-\mathrm{g}$ increment in milk/dairy 
Table 2. Multivariable-adjusted mean fetuin-A concentrations $(95 \% \mathrm{Cl})$ by quintiles of energy intake or energy-providing nutrient intake in 558 men and women who participated in the Bavarian Food Consumption Survey II

\begin{tabular}{|c|c|c|c|c|c|c|c|c|c|c|c|c|c|}
\hline & \multicolumn{10}{|c|}{ Quintiles of energy intake or energy-providing nutrients } & \multirow{3}{*}{\multicolumn{2}{|c|}{$\begin{array}{l}\text { Continuous } \\
\text { estimate* }\end{array}$}} & \multirow[b]{3}{*}{$P_{\text {trend }}$} \\
\hline & \multicolumn{2}{|c|}{ Quintile 1} & \multicolumn{2}{|c|}{ Quintile 2} & \multicolumn{2}{|c|}{ Quintile 3} & \multicolumn{2}{|c|}{ Quintile 4} & \multicolumn{2}{|c|}{ Quintile 5} & & & \\
\hline & $\begin{array}{l}\text { Mean } \\
\text { fetuin-A }\end{array}$ & $95 \% \mathrm{Cl}$ & $\begin{array}{l}\text { Mean } \\
\text { fetuin-A }\end{array}$ & $95 \% \mathrm{Cl}$ & $\begin{array}{l}\text { Mean } \\
\text { fetuin-A }\end{array}$ & $95 \% \mathrm{Cl}$ & $\begin{array}{l}\text { Mean } \\
\text { fetuin-A }\end{array}$ & $95 \% \mathrm{Cl}$ & $\begin{array}{l}\text { Mean } \\
\text { fetuin-A }\end{array}$ & $95 \% \mathrm{Cl}$ & & & \\
\hline \multicolumn{14}{|l|}{ Energy\|l } \\
\hline $\begin{array}{l}\text { Age-adjusted and } \\
\text { sex-adjusted }\end{array}$ & 303 & 293,313 & 317 & 307,327 & 301 & 291,310 & 317 & 307,327 & 313 & 302,323 & $2 \cdot 7$ & $-1 \cdot 4,6 \cdot 8$ & 0.31 \\
\hline Multivariable† & 301 & 291,311 & 316 & 306,327 & 301 & 291,311 & 318 & 308,327 & 312 & 302,323 & 3.3 & $-0.8,7.4$ & 0.20 \\
\hline $\begin{array}{l}\text { Multivariable† plus } \\
\text { body fatness } \uparrow\end{array}$ & 300 & 290,310 & 316 & 305,326 & 301 & 291,311 & 318 & 308,327 & 313 & 302,323 & 3.7 & $-0.5,7 \cdot 8$ & $0 \cdot 16$ \\
\hline \multicolumn{14}{|l|}{$\begin{array}{l}\text { Fat (percentage of } \\
\text { energy) }\end{array}$} \\
\hline $\begin{array}{l}\text { Age- and sex- } \\
\text { adjusted }\end{array}$ & 305 & 295,315 & 306 & 296,315 & 311 & 301,320 & 315 & 304,325 & 315 & 305,325 & 3.0 & $-0.1,6 \cdot 2$ & 0.09 \\
\hline $\begin{array}{l}\text { Multivariable } \\
\text { (carbohydrate } \\
\text { substitution } \\
\text { model)‡ }\end{array}$ & 306 & 295,317 & 307 & 297,318 & 310 & 301,320 & 313 & 302,323 & 312 & 302,323 & $1 \cdot 8$ & $-1 \cdot 6,5 \cdot 2$ & 0.33 \\
\hline $\begin{array}{l}\text { Multivariableł plus } \\
\text { body fatness }\end{array}$ & 306 & 296,317 & 308 & 298,318 & 311 & 302,321 & 312 & 301,322 & 311 & 300,321 & 1.6 & $-1 \cdot 8,4 \cdot 9$ & 0.47 \\
\hline \multicolumn{14}{|l|}{$\begin{array}{l}\text { Carbohydrates } \\
\text { (percentage of } \\
\text { energy) }\end{array}$} \\
\hline $\begin{array}{l}\text { Age- and sex- } \\
\text { adjusted }\end{array}$ & 315 & 303,326 & 306 & 296,315 & 307 & 298,317 & 312 & 302, 322 & 311 & 301,321 & 0.3 & $-2 \cdot 7,3 \cdot 4$ & 0.89 \\
\hline $\begin{array}{l}\text { Multivariable (fat } \\
\text { substitution } \\
\text { model) } \ddagger\end{array}$ & 320 & 308,331 & 307 & 297,318 & 306 & 296,316 & 309 & 298,319 & 306 & 295,316 & $-1 \cdot 8$ & $-5 \cdot 2,1 \cdot 6$ & 0.14 \\
\hline $\begin{array}{l}\text { Multivariableł plus } \\
\text { body fatness }\end{array}$ & 319 & 307,330 & 307 & 297,317 & 306 & 297,316 & 309 & 299,319 & 306 & 296,316 & $-1 \cdot 6$ & $-4 \cdot 9,1 \cdot 8$ & $0 \cdot 17$ \\
\hline \multicolumn{14}{|l|}{$\begin{array}{l}\text { Protein (percentage } \\
\text { of energy) }\end{array}$} \\
\hline $\begin{array}{l}\text { Age- and sex- } \\
\text { adjusted }\end{array}$ & 319 & 309,329 & 309 & 299,320 & 304 & 295,312 & 307 & 297,317 & 311 & 301,322 & $-2 \cdot 4$ & $-10 \cdot 5,5 \cdot 6$ & 0.31 \\
\hline $\begin{array}{l}\text { Multivariable } \\
\text { (carbohydrate } \\
\text { substitution } \\
\text { model)§ }\end{array}$ & 319 & 308,330 & 308 & 298,318 & 304 & 295,313 & 306 & 296,317 & 312 & 301,323 & $-2 \cdot 2$ & $-10 \cdot 6,6 \cdot 1$ & 0.35 \\
\hline $\begin{array}{l}\text { Multivariable§ plus } \\
\text { body fatness } \mathbb{}\end{array}$ & 321 & 310,332 & 309 & 298,319 & 303 & 294,312 & 306 & 296,316 & 309 & 298,320 & -4.9 & $-13 \cdot 3,3 \cdot 6$ & $0 \cdot 11$ \\
\hline
\end{tabular}

* Increments are $2092 \mathrm{~kJ} / \mathrm{d}$ (500 kcal/d for total energy); $5 \%$ of energy for fat, carbohydrates and protein.

$\dagger$ Multivariable adjusted for age, sex, smoking status, social status, physical activity, alcohol intake (non-drinker or g/d).

¥ Multivariable adjusted for age, sex, smoking status, social status, physical activity, energy intake, alcohol intake (non-drinker or \% of energy) and protein intake (\% of energy).

$\S$ Multivariable adjusted for age, sex, smoking status, social status, physical activity, energy intake, alcohol intake (non-drinker or \% of energy), fat intake (\% of energy).

II Energy intake refers to non-alcohol energy intake.

II Plus body fatness refers to additional adjustment for BMI and BMI-adjusted waist circumference residuals.

Table 3. Multivariable-adjusted mean fetuin-A concentrations $(95 \% \mathrm{Cl})$ by categories of alcohol consumption in 558 men and women who participated in the Bavarian Food Consumption Survey II

\begin{tabular}{|c|c|c|c|c|c|c|c|c|c|c|c|}
\hline & \multicolumn{10}{|c|}{ Alcohol intake categories } & \multirow[b]{3}{*}{$P_{\text {trend }}$} \\
\hline & \multicolumn{2}{|c|}{ Non-drinker } & \multicolumn{2}{|c|}{$<5 \mathrm{~g} / \mathrm{d}$} & \multicolumn{2}{|c|}{5 to $<15 \mathrm{~g} / \mathrm{d}$} & \multicolumn{2}{|c|}{15 to $<30 \mathrm{~g} / \mathrm{d}$} & \multicolumn{2}{|c|}{$\geq 30 \mathrm{~g} / \mathrm{d}$} & \\
\hline & $\begin{array}{l}\text { Mean } \\
\text { fetuin-A }\end{array}$ & $95 \% \mathrm{Cl}$ & $\begin{array}{l}\text { Mean } \\
\text { fetuin-A }\end{array}$ & $95 \% \mathrm{Cl}$ & $\begin{array}{l}\text { Mean } \\
\text { fetuin-A }\end{array}$ & $95 \% \mathrm{Cl}$ & $\begin{array}{l}\text { Mean } \\
\text { fetuin-A }\end{array}$ & $95 \% \mathrm{Cl}$ & $\begin{array}{l}\text { Mean } \\
\text { fetuin-A }\end{array}$ & $95 \% \mathrm{Cl}$ & \\
\hline$n$ & 86 & & 161 & & 134 & & 94 & & 83 & & \\
\hline Age- and sex-adjusted & 325 & 314,335 & 311 & 302,319 & 314 & 305,323 & 302 & 291,313 & 297 & 285,308 & 0.004 \\
\hline Multivariable adjusted* & 326 & 315,337 & 311 & 302,320 & 313 & 303,323 & 302 & 290,313 & 295 & 282,307 & 0.003 \\
\hline $\begin{array}{l}\text { Multivariable adjusted* plus } \\
\text { body fatness } \dagger\end{array}$ & 324 & 313,335 & 311 & 302,320 & 314 & 304,323 & 303 & 292,314 & 293 & 281,306 & 0.003 \\
\hline
\end{tabular}

* Multivariable adjusted for age, sex, smoking status, social status, physical activity and energy intake (excluding energy from alcoholic beverages).

$\dagger$ Plus body fatness refers to additional adjustment for BMI and BMI-adjusted waist circumference residuals. 
Table 4. Multivariable-adjusted mean fetuin-A concentrations ( $95 \% \mathrm{Cl}$ ) by quintiles or categories of major food groups in 558 men and women who participated in the Bavarian Food Consumption Survey II

\begin{tabular}{|c|c|c|c|c|c|c|c|c|c|c|c|c|c|}
\hline & \multicolumn{10}{|c|}{ Quintiles or categories of food intake } & \multirow{3}{*}{\multicolumn{2}{|c|}{ Continuous estimate $§ \S$}} & \multirow[b]{3}{*}{$P_{\text {trend }}$} \\
\hline & \multicolumn{2}{|c|}{ Quintile 1} & \multicolumn{2}{|c|}{ Quintile 2} & \multicolumn{2}{|c|}{ Quintile 3} & \multicolumn{2}{|c|}{ Quintile 4} & \multicolumn{2}{|c|}{ Quintile 5} & & & \\
\hline & Mean fetuin-A & $95 \% \mathrm{Cl}$ & Mean fetuin-A & $95 \% \mathrm{Cl}$ & Mean fetuin-A & $95 \% \mathrm{Cl}$ & Mean fetuin-A & $95 \% \mathrm{Cl}$ & Mean fetuin-A & $95 \% \mathrm{Cl}$ & & & \\
\hline Age- and sex-adjusted & 320 & 310,330 & 312 & 301,323 & 301 & 291,311 & 307 & 298,317 & 310 & 300,320 & $-2 \cdot 5$ & $-7 \cdot 5,2 \cdot 44$ & 0.22 \\
\hline Multivariable† & 320 & 309,331 & 312 & 302,323 & 301 & 291,312 & 307 & 297,317 & 308 & 298,318 & $-3 \cdot 3$ & $-8 \cdot 2,1 \cdot 64$ & 0.13 \\
\hline Multivariable $\nmid$ plus body fatness $\ddagger$ & 320 & 309,331 & 313 & 302,323 & 301 & 291,311 & 307 & 297,317 & 308 & 298,318 & $-3 \cdot 2$ & $-8 \cdot 1,1 \cdot 70$ & 0.13 \\
\hline Fruit & & & & & & & & & & & & & \\
\hline Age- and sex-adjusted & 314 & 304,325 & 305 & 294,316 & 314 & 304,324 & 310 & 300,320 & 307 & 298,316 & -1.5 & $-6 \cdot 3,3 \cdot 36$ & 0.52 \\
\hline Age- and sex-adjusted & 318 & 309,328 & 310 & 300,319 & 314 & 304,324 & 304 & 293,316 & 304 & 294,314 & $-4 \cdot 6^{*}$ & $-8.8,-0.43$ & $0.05^{\star}$ \\
\hline Multivariable† & 320 & 309,330 & 310 & 301,319 & 314 & 304,325 & 304 & 293,315 & 302 & 291,312 & $-5 \cdot 9^{*}$ & $-9.9,-1.8$ & $0.02^{*}$ \\
\hline Multivariable $\nmid$ plus body fatness $\ddagger$ & 320 & 309,330 & 311 & 302,320 & 313 & 302,323 & 304 & 293,315 & 303 & 292,313 & $-5 \cdot 6^{\star}$ & $-9 \cdot 6,-1 \cdot 5$ & $0.02^{*}$ \\
\hline Unprocessed red meat & & & & & & & & & & & & & \\
\hline Age- and sex-adjusted & 313 & 306,320 & 329 & 308,351 & 307 & 297,317 & 308 & 299,318 & 307 & 297,318 & -0.47 & $-6 \cdot 0,5.00$ & 0.36 \\
\hline Multivariable & 313 & 305,321 & 330 & 309, 351 & 305 & 295,315 & 307 & 297,317 & 308 & 296,319 & -0.46 & $-6 \cdot 1,5 \cdot 13$ & 0.38 \\
\hline Multivariable plus body fatness & 313 & 306,321 & 328 & 308,349 & 305 & 296,315 & 308 & 298,318 & 306 & 295,317 & $-1 \cdot 1$ & $-6 \cdot 7,4 \cdot 40$ & 0.27 \\
\hline Processed meat & & & & & & & & & & & & & \\
\hline Poultry & & & & & & & & & & & & & \\
\hline$n$ & 382 & & 76 & & 90 & & & & & & & & \\
\hline Age- and sex-adjusted & 311 & 306,316 & 307 & 294,319 & 309 & 298,321 & & & & & -0.55 & $-4.7,3.61$ & 0.72 \\
\hline Multivariable† & 310 & 304,316 & 307 & 295,319 & 310 & 298,322 & & & & & -0.15 & $-4 \cdot 4,4 \cdot 14$ & 0.91 \\
\hline Multivariable plus body fatnessł & 310 & 304,316 & 306 & 294,318 & 310 & 298, 322 & & & & & -0.27 & $-4.5,4.00$ & 0.89 \\
\hline Fish & & & & & & & & & & & & & \\
\hline$n$ & 365 & & 90 & & 93 & & & & & & & & \\
\hline Age- and sex-adjusted & 311 & 305,316 & 305 & 295,316 & 313 & 302,324 & & & & & 1.66 & $-2 \cdot 0,5 \cdot 27$ & 0.78 \\
\hline Multivariable† & 310 & 304,316 & 305 & 294,315 & 313 & 302,325 & & & & & 1.87 & $-1 \cdot 9,5 \cdot 62$ & 0.66 \\
\hline Multivariable† plus body fatnessł & 310 & 304,316 & 305 & 295,315 & 312 & 301,324 & & & & & -1.56 & $-2 \cdot 2,5 \cdot 28$ & 0.80 \\
\hline
\end{tabular}

* Statistically significant results $(P<0.05)$.

† Multivariable adjusted for age, sex, smoking status, social status, physical activity, alcohol intake (non-drinker or g/d) and non-alcohol energy intake.

‡ Plus body fatness refers to additional adjustment for BMI and BMI-adjusted waist circumference residuals.

$\S$ Continuous estimates were calculated per portion. Portion sizes based on approximate standard deviations: vegetables, $100 \mathrm{~g} / \mathrm{d}$; fruit, $150 \mathrm{~g} / \mathrm{d}$; dairy products, $150 \mathrm{~g} / \mathrm{d}$; unprocessed red meat, $50 \mathrm{~g} / \mathrm{d}$; processed meat, $50 \mathrm{~g} / \mathrm{d}$; poultry,

$30 \mathrm{~g} / \mathrm{d}$; fish, $30 \mathrm{~g} / \mathrm{d}$. 
products per d was associated with $5 \cdot 6(95 \% \mathrm{CI}-9 \cdot 6,-1 \cdot 5) \mu \mathrm{g} / \mathrm{ml}$ lower fetuin-A. This inverse association was slightly attenuated after additional adjustment for protein $(-4.9 \mu \mathrm{g} / \mathrm{ml}, 95 \%$ CI $-9 \cdot 1$, $-0.7 \mu \mathrm{g} / \mathrm{ml}$ ), but it was not substantially altered after additional adjustment for fat $(-5.4 \mu \mathrm{g} / \mathrm{ml}, 95 \% \mathrm{CI}-9.4,-1.4 \mu \mathrm{g} / \mathrm{ml})$ or $\mathrm{Ca}$ intake $(-5.4 \mu \mathrm{g} / \mathrm{ml}, 95 \%$ CI $-11 \cdot 2,0.4 \mu \mathrm{g} / \mathrm{ml})$. Milk, yogurt and cheese were also individually inversely associated with fetuin-A (milk per $100 \mathrm{~g} / \mathrm{d}-3.3 \mu \mathrm{g} / \mathrm{ml}, 95 \% \mathrm{CI}-6.5,-0.2 \mu \mathrm{g} / \mathrm{ml}$; yogurt per $50 \mathrm{~g} / \mathrm{d}-2.0 \mu \mathrm{g} / \mathrm{ml}, 95 \% \mathrm{CI}-5 \cdot 6,1.7 \mu \mathrm{g} / \mathrm{ml}$; cheese per $30 \mathrm{~g} / \mathrm{d}$ $-2 \cdot 4 \mu \mathrm{g} / \mathrm{ml}, 95 \% \mathrm{CI}-7 \cdot 5,2 \cdot 8 \mu \mathrm{g} / \mathrm{ml})$. However, the inverse association between milk and dairy product intake and fetuin-A concentrations was statistically nonsignificant after accounting for the false-discovery rate (adjusted $P_{\text {trend }} 0 \cdot 18$ ). Fetuin-A concentrations were not associated with dietary intake of vegetables, fruit, unprocessed red meat, processed meat, poultry or fish.

\section{Discussion}

To our knowledge, this is the first observational study comprehensively investigating dietary determinants of fetuin-A. Total energy intake and energy-providing nutrients were not clearly associated with fetuin-A concentrations. We observed that alcohol intake was associated with lower fetuin-A concentrations. Among the major food groups, higher dietary intake of milk/dairy products was associated with lower circulating fetuin-A, but this association was not statistically significant after correction for multiple hypothesis testing. Dietary intakes of fruit, vegetables, meat or fish were not associated with fetuin-A concentrations. Because of the described association between fetuin-A and obesity, insulin resistance, diabetes and coronary heart disease, modulation of fetuin-A concentration by dietary guidance may be of public health relevance.

The here observed inverse association between alcohol intake and fetuin-A is largely supported by the existing literature: Univariable inverse associations between alcohol intake and fetuin-A have been observed in several epidemiological studies $^{(4,7,9,23-25)}$. Similar to our investigation, higher alcohol consumption was associated with lower fetuin-A concentrations in women participating in the Nurses' Health Study after adjustment for demographic information and lifestyle variables including $\mathrm{BMI}^{(12)}$. Furthermore, post hoc analyses of three randomised cross-over trials on alcohol intake revealed that moderate alcohol consumption decreased fetuin-A in men, although no significant association was observed in women ${ }^{(26)}$. To date, the physiological mechanisms that may explain the inverse association between alcohol intake and fetuin-A remain unclear.

We also observed that higher milk/dairy product intake was statistically significantly associated with lower fetuin-A, although statistical significance was lost after accounting for the falsediscovery rate. The inverse association was slightly attenuated after additional adjustment for protein, suggesting that protein may partly explain the association. However, adjustment for other nutrients found in dairy products, such as fat or Ca, did not alter the associations remarkably. Several epidemiological studies have observed an inverse association between dairy product consumption and presence of the metabolic syndrome ${ }^{(27,28)}$. In a prospective study among young adults, inverse associations between dairy product consumption and the development of obesity and insulin resistance were observed ${ }^{(29)}$. Furthermore, a few intervention studies have shown that dairy product consumption is associated with improved insulin sensitivity ${ }^{(30)}$. Considering the role of fetuin-A in the insulin signalling pathway ${ }^{(31)}$ - that is, induction of insulin resistance through inhibition of insulin-receptor tyrosine kinase ${ }^{(32)}$ - it is conceivable that fetuin-A may have a mediating role in the association between dairy product consumption and improved insulin sensitivity. This warrants further exploration in prospective studies.

Several limitations of this study should be noted. First of all, because of the cross-sectional study design in which the dietary exposure and the biomarker outcome both were assessed within a short time period, it is difficult to determine the direction of observed associations, and we cannot make any causal inferences. We hypothesised that dietary factors would influence fetuin-A concentrations. It appears unlikely that fetuin-A concentrations directly influence dietary habits, but we cannot exclude that, for example, existing fatty liver disease reflected by high fetuin-A concentrations may have led to a change in dietary habits, because of dietary recommendations given by general practitioners. In addition, although through multivariable adjustment we tried to control for potential confounding as completely as possible, residual confounding cannot be excluded. We also cannot exclude the possibility that storage of plasma samples at $-80^{\circ} \mathrm{C}$ for approximately 10 years may have affected fetuin-A concentrations. However, any such impact on fetuin-A measurement is unlikely to be differential according to participant's dietary intake, and thus it is unlikely to have introduced systematic bias. Furthermore, the absolute fetuin-A concentrations in our study were comparable to concentrations that have been observed in other observational studies in Germany ${ }^{(1,7)}$. In our study, habitual dietary intake was assessed with three 24-h dietary recalls involving detailed quantification of consumed foods including composition of mixed meals. As with all self-reported methods, the dietary assessment by three 24-h recalls is prone to measurement error, as it depends on the participants' memory and ability to recall their diet. In addition, because the 24-h recall is an open-ended instrument, the interviewer is also a potential source of bias. However, the training of interviewers and the high standardisation of the 24-h recalls conducted with EPIC-soft limits this source of bias ${ }^{(15)}$. The here applied number of three $24-\mathrm{h}$ recalls has been shown to be sufficient for estimating total energy intake with energy intake assessment using the doubly labelled water (DLW) as the reference method ${ }^{(33)}$. However, misclassification of diet, in particular under-reporting of energy intake, remains a concern when relating self-reported dietary intake to health outcomes. Under-reporting of energy intake has been reported to be more prevalent in individuals with a high BMI and in women ${ }^{(20,21)}$. A recent pooled analysis of five DLW validation studies demonstrated that under-reporting of energy intake was approximately $10 \%$ with three averaged 24-h recalls compared with $30 \%$ with $\mathrm{FFQ}^{(34)}$. Although dietary intake is reported more accurately with multiple 24-h recalls than with FFQ, under-reporting of energy intake had a role also in the present study: by comparing the reported energy intake with the estimated basal metabolic rate, we identified a 
proportion of under-reporters of energy intake ( $n 46,8 \%)$ in our sample. In the analysis of energy intake in relation to fetuin-A concentrations, continuous estimates were slightly stronger after exclusion of under-reporters, and statistically significant only in lean study participants and in men. These observations point to the problem of misreporting. Thus, the analysis of energy intake in relation to fetuin-A should be interpreted with caution. Overall, we expect misreporting of diet in our study to be non-differential - that is, misclassification independent of fetuin-A concentrations - which may have biased observed associations towards a null association. A further limitation of our study is related to the generalisability of our findings. Although the BVS II was designed as a representative study, the findings observed here can be generalised to the adult Bavarian population only with caution, as the overall participation of adult study participants who also provided blood samples was $46 \%(71 \% \times 65 \%)$, thus compromising the representativeness of our study sample. Whether our findings may be generalised to other populations warrants further investigation, but we expect that the associations found in our study should be comparable in populations with similar characteristics as in our study.

In conclusion, in this comprehensive investigation of dietary determinants of fetuin-A, we observed that higher consumption of alcohol and dietary intake of milk/dairy products were associated with lower fetuin-A concentrations. These observations warrant confirmation by further observational studies or controlled feeding intervention studies. Nevertheless, our findings provide a first suggestion that fetuin-A concentrations may be influenced by targeted dietary interventions. Considering the role of fetuin-A in the development of obesity, insulin resistance, diabetes and coronary heart disease, this may be of direct public health importance. Whether previously observed associations between dietary intake and health outcomes are mediated by fetuin-A requires exploration in prospective cohort studies.

\section{Acknowledgements}

The authors acknowledge the cooperation of all study participants. They also thank Georg Karg, Kurt Gedrich and Stefanie Himmerich for their major contribution in the setup and conduct of the study.

This work was supported by funds of the Bavarian Ministry of Environment, Health and Consumer Protection and the Kurt-Eberhard-Bode-Stiftung.

The authors' contributions were as follows: K. N., T. P. and J. L. contributed to the conception and design of the research; K. N. performed statistical analyses, interpreted the data and wrote the paper; J. L. was responsible for the concept and design of the BVS II, as well as the acquisition of data; J. J. was responsible for the measurement of fetuin-A in plasma samples; all authors critically appraised the manuscript and approved the final version.

There are no conflicts of interest.

\section{Supplementary material}

For Supplementary material/s referred to in this article, please visit http://dx.doi.org/10.1017/S0007114515002639

\section{References}

1. Stefan N, Hennige AM, Staiger H, et al. (2006) Alpha2-Heremans-Schmid glycoprotein/fetuin-A is associated with insulin resistance and fat accumulation in the liver in humans. Diabetes Care 29, 853-857.

2. Chatterjee P, Seal S, Mukherjee S, et al. (2013) Adipocyte fetuin-A contributes to macrophage migration into adipose tissue and polarization of macrophages. J Biol Chem 288, 28324-28330.

3. Ix JH, Shlipak MG, Brandenburg VM, et al. (2006) Association between human fetuin-A and the metabolic syndrome: data from the Heart and Soul Study. Circulation 113, 1760-1767.

4. Stefan N, Fritsche A, Weikert C, et al. (2008) Plasma fetuin-A levels and the risk of type 2 diabetes. Diabetes 57, 2762-2767.

5. Auberger P, Falquerho L, Contreres JO, et al. (1989) Characterization of a natural inhibitor of the insulin receptor tyrosine kinase: cDNA cloning, purification, and antimitogenic activity. Cell 58, 631-640.

6. Mathews ST, Singh GP, Ranalletta M, et al. (2002) Improved insulin sensitivity and resistance to weight gain in mice null for the Ahsg gene. Diabetes 51, 2450-2458.

7. Weikert C, Stefan N, Schulze MB, et al. (2008) Plasma fetuin-a levels and the risk of myocardial infarction and ischemic stroke. Circulation 118, 2555-2562.

8. Thakkinstian A, Chailurkit L, Warodomwichit $\mathrm{D}$, et al. (2013) Causal relationship between body mass index and fetuin-A level in the asian population: a bidirectional mendelian randomization study. Clin Endocrinol (Oxf) 81, 197-203.

9. Ix JH, Biggs ML, Mukamal KJ, et al. (2012) Association of fetuin-A with incident diabetes mellitus in community-living older adults: the Cardiovascular Health Study. Circulation 125, 2316-2322.

10. Choi KM, Han KA, Ahn HJ, et al. (2013) The effects of caloric restriction on fetuin-A and cardiovascular risk factors in rats and humans: a randomized controlled trial. Clin Endocrinol (Oxf) 79, 356-363.

11. Montonen J, Boeing H, Fritsche A, et al. (2012) Consumption of red meat and whole-grain bread in relation to biomarkers of obesity, inflammation, glucose metabolism and oxidative stress. Eur J Nutr 52, 337-345.

12. Ley SH, Sun Q, Jimenez MC, et al. (2014) Association between alcohol consumption and plasma fetuin-A and its contribution to incident type 2 diabetes in women. Diabetologia $\mathbf{5 7}$, 93-101.

13. Himmerich S, Gedrich K \& Karg G (2003) Bayerische Verzebrsstudie (BVS) II (Bavarian Food Consumption Survey (BVS) II). Munich, Germany: Bayerischen Staatsministeriums fuer Umwelt, Gesundheit und Verbraucherschutz.

14. Slimani N, Deharveng G, Charrondiere RU, et al. (1999) Structure of the standardized computerized 24-h diet recall interview used as reference method in the 22 centers participating in the EPIC project. European prospective investigation into cancer and nutrition. Comput Methods Programs Biomed 58, 251-266.

15. Slimani N, Ferrari P, Ocke M, et al. (2000) Standardization of the 24-hour diet recall calibration method used in the European prospective investigation into cancer and nutrition (EPIC): general concepts and preliminary results. Eur J Clin Nutr 54, 900-917.

16. Dehne LI, Klemm C, Henseler G, et al. (1999) The German food code and nutrient data base (BLS II.2). Eur J Epidemiol 15, 355-359.

17. White H (1980) A heteroskedasticity-consistent covariance matrix estimator and a direct test for heteroskedasticity. Econometrica 48, 817-838. 
18. Pai JK, Hankinson SE, Thadhani R, et al. (2006) Moderate alcohol consumption and lower levels of inflammatory markers in US men and women. Atherosclerosis 186, 113-120.

19. Benjamini Y, Drai D, Elmer G, et al. (2001) Controlling the false discovery rate in behavior genetics research. Behav Brain Res 125, 279-284.

20. Ferrari P, Slimani N, Ciampi A, et al. (2002) Evaluation of under- and overreporting of energy intake in the 24-hour diet recalls in the European Prospective Investigation into Cancer and Nutrition (EPIC). Public Health Nutr 5 , $1329-1345$.

21. Heerstrass DW, Ocke MC, Bueno-de-Mesquita HB, et al. (1998) Underreporting of energy, protein and potassium intake in relation to body mass index. Int J Epidemiol 27, 186-193.

22. World Health Organization (1985) Energy and protein requirements. Report of a joint $\mathrm{FAO} / \mathrm{WHO} / \mathrm{UNU}$ Expert Consultation. World Health Organ Tech Rep Ser 724, 1-206.

23. Jensen MK, Bartz TM, Mukamal KJ, et al. (2013) Fetuin-A, type 2 diabetes, and risk of cardiovascular disease in older adults: the Cardiovascular Health Study. Diabetes Care 36, 12221228 .

24. Laughlin GA, Barrett-Connor E, Cummins KM, et al. (2013) Sex-specific association of fetuin-A with type 2 diabetes in older community-dwelling adults: the Rancho Bernardo Study. Diabetes Care 36, 1994-2000.

25. Obuchi A, Adachi H, Enomoto M, et al. (2014) High plasma fetuin-A levels are associated with metabolic syndrome among males but not females in a Japanese general population. Diabetes Res Clin Pract 106, 128-135.
26. Joosten MM, Schrieks IC \& Hendriks HF (2014) Effect of moderate alcohol consumption on fetuin-A levels in men and women: post-hoc analyses of three open-label randomized crossover trials. Diabetol Metab Syndr 6, 24.

27. Elwood PC, Pickering JE \& Fehily AM (2007) Milk and dairy consumption, diabetes and the metabolic syndrome: the Caerphilly prospective study. J Epidemiol Community Health 61, 695-698.

28. Van Meijl LE, Vrolix R \& Mensink RP (2008) Dairy product consumption and the metabolic syndrome. Nutr Res Rev 21, 148-157.

29. Pereira MA, Jacobs DR Jr., Van Horn L, et al. (2002) Dairy consumption, obesity, and the insulin resistance syndrome in young adults: the CARDIA Study. JAMA 287, 2081-2089.

30. Turner KM, Keogh JB \& Clifton PM (2014) Dairy consumption and insulin sensitivity: a systematic review of short- and longterm intervention studies. Nutr Metab Cardiovasc Dis 25, 3-8.

31. Srinivas PR, Wagner AS, Reddy LV, et al. (1993) Serum alpha 2HS-glycoprotein is an inhibitor of the human insulin receptor at the tyrosine kinase level. Mol Endocrinol 7, 1445-1455.

32. Hennige AM, Staiger H, Wicke C, et al. (2008) Fetuin-A induces cytokine expression and suppresses adiponectin production. PLOS ONE 3, e1765.

33. Ma Y, Olendzki BC, Pagoto SL, et al. (2009) Number of 24-hour diet recalls needed to estimate energy intake. Ann Epidemiol 19, 553-559.

34. Freedman LS, Commins JM, Moler JE, et al. (2014) Pooled results from 5 validation studies of dietary self-report instruments using recovery biomarkers for energy and protein intake. $\mathrm{Am} \mathrm{J}$ Epidemiol 180, 172-188. 